Cristian Paúl Espinoza-Espinoza; Darwin Gabriel García-Herrera; Cristian Andrés Erazo-Álvarez; Juan Carlos Erazo-Álvarez

http://dx.doi.org/10.35381/e.k.v3i1.1015

\title{
Pedagogía del ciberespacio un reto para los docentes
}

Cyberspace pedagogy a challenge for teachers

\author{
Cristian Paúl Espinoza-Espinoza \\ cristianpaul.espinoza@est.ucacue.edu.ec \\ Universidad Católica de Cuenca, Azogues \\ Ecuador \\ https://orcid.org/0000-0002-4270-9785 \\ Darwin Gabriel García-Herrera \\ dggarciah@ucacue.edu.ec \\ Universidad Católica de Cuenca, Azogues \\ Ecuador \\ https://orcid.org/0000-0001-6813-8100 \\ Cristian Andrés Erazo-Álvarez \\ cristianerazo@ucacue.edu.ec \\ Universidad Católica de Cuenca, Cuenca \\ Ecuador \\ https://orcid.org/0000-0001-8746-4788 \\ Juan Carlos Erazo-Álvarez \\ jcerazo@ucacue.edu.ec \\ Universidad Católica de Cuenca, Cuenca \\ Ecuador \\ https://orcid.org/0000-0001-6480-2270
}

Recepción: 10 agosto 2020

Revisado: 25 septiembre 2020

Aprobación: 15 octubre 2020

Publicación: 01 noviembre 2020 


\title{
RESUMEN
}

El objetivo fue analizar la incidencia de la pedagogía del ciberespacio durante la transición de un cambio de una educación en modalidad presencial a una educación en modalidad virtual en donde el docente es el guía y mediador para la incorporación de las TIC en los espacios virtuales de aprendizaje. La metodologia utilizada fue de carácter no experimental con un enfoque metodologico mixto y de cohorte transversal. Los docentes además de adquirir y adaptar metodologías activas en la enseñanza para el nuevo entorno de aprendizaje tienen que desarrollar habilidades y destrezas digitales que ayuden a incentivar a sus estudiantes a dar un buen uso a la tecnología no solo dentro y fuera de las clases. El personal docente igual que sus estudiantes deben aprender de competencias digitales, pero aún más orientarlas de mejor manera a las necesidades que plantea esta nueva sociedad del conocimiento.

Descriptores: Innovación educacional; tendencia educacional; desarrollo de la educación. (Palabras tomadas del Tesauro UNESCO).

\begin{abstract}
The objective was to analyze the incidence of cyberspace pedagogy during the transition from a change from face-to-face education to virtual education in which the teacher is the guide and mediator for the incorporation of ICT in virtual learning spaces. The methodology used was non-experimental with a mixed methodological approach and a cross-sectional cohort. Teachers, in addition to acquiring and adapting active teaching methodologies for the new learning environment, have to develop digital skills and abilities that help encourage their students to make good use of technology not only inside and outside of class. The teaching staff, like their students, must learn digital skills, but even more orient them in a better way to the needs posed by this new knowledge society.
\end{abstract}

Descriptors: Educational innovations; educational trends; educational development. (Words taken from the UNESCO Thesaurus). 


\section{INTRODUCCIÓN}

La presente investigación esta orientada hacia la pedagogía del ciberespacio como un reto innovador para los docentes, podemos decir que la pedagogía es la ciencia que estudia los métodos y formas de enseñar, el reto en la actualidad es lograr combinar a la pedagogía con las tecnologías, ya que en la actualidad es una necesidad primordial en la educación. Por motivos de Covid a nivel mundial se dieron varios cambios que afectaron a la sociedad en general, especialmente en el ámbito de educación donde paso de una educación presencial a una educación de forma virtual, trajo consigo muchos problemas especialmente en los países sub desarrollados o en vías de desarrollo, entre ellos el nuestro, que no estuvo preparado para afrontar las necesidades que nos exige la educación el día de hoy.

Los docentes necesitan adquirir competencias instrumentales para la utilización de las tecnologías, pero sobre todo necesitan la adquisición y empoderamiento de competencias pedagógicas para optimizar todos estos recursos TIC dentro de los espacios virtuales en donde trabaje el docente (Mirete, 2010). La introducción de las tecnologías como parte del cambio al modelo educativo actual demanda un profundo análisis para la incorporación de TIC mediante un proceso de alfabetización docente en ámbito digital, el mismo que debe estar en un proceso continuó de capacitación y actualización según las necesidades que se presenten dentro del contexto educativo.La pedagogía del ciberespacio es la clave fundamental para el éxito de incorporación de TIC en la educación.

En los últimos tiempos la educación demanda un cambio en la forma de dar y recibir clases a un más en la función que debe asumir el docente dentro del ciberespacio se le pide que se convierta en un mediador, que atienda a la diversidad, que logre desarrollar en sus estudiantes un pensamiento crítico, que utilice nuevas metodologías, tecnologías, que innove, que conozca su entorno y el contexto cultural concreto en el que se desenvuelve y sobretodo alcance niveles importantes de motivación en los estudiantes. 
Pensar en un verdadero cambio desde la actitud hasta llegar a un cambio en la formación pedagógica en el uso y manejo de las tecnologías, esta propuesta novedosa para implementarla en los docentes no ha de ser aquella que lo transforme al docente en un técnico, sino que ayude al proceso de selección de contenidos que le facilite el proceso de enseñanza con una orientación pedagógica acorde a las necesidades reales de la educación. Para desarrollar estas tecnologías y ser implementadas dentro de educación primero deberán adoptar un ámbito pedagógico para generar procesos de aprendizajes significativos, por lo tanto no se puede negar que el desarrollo de la competencia digital, tiene mucha relevancia en la formación integral para un aprendizaje permanente dentro del contexto actual del ciberespacio como ambiente educativo, precisamente, esta responsabilidad recae en la labor que desempeñan los docentes (Gisbert-Cervera \& Lázaro-Cantabrana, 2015).

Los espacios virtuales de aprendizaje presentan varias alternativas para el desarrollo del proceso de enseñanza, estos pueden ser de forma sincrónica o asincrónica facilitando el acceso a la información en red, pero es importante recalcar el rol del docente, es el guía dentro de este proceso de selección de la información, debe elegir de entre un sinnúmero de recursos y herramientas digitales, cual se adapte a las necesidades reales de lo estudiantes, que beneficio y utilidad se les pueda dar estableciendo así una nueva forma de dar y de recibir clases.

Por lo antes expueto, el presente trabajo tiene como finalidad analizar la incidencia de la pedagogía del ciberespacio durante esta transición de una educacion precensial a una educacion de forma virtual en donde el docente es el guia y mediador para la incorpocación de las TIC en los espacios virtales de aprendizaje y establecer alternativas de aplicación en la educacion ecuatoriana. 


\section{Referencial teórico}

La formación de competencias digitales para docentes en educación tomando como referentes algunos programas como habilidades digitales para todos, el cual se base fundamental en la importancia y trascendencia de la formación digital como nueva propuesta para hacer frente a las expectativas y retos que plantea esta nueva era, al introducir las TIC a la práctica docente. Así, esta investigación realiza una afirmación de que de la formación docente es un elemento fundamental en el desarrollo de los proyectos educativos, es la forma en la que se promueve el desarrollo de competencias digitales entre los docentes es la se busca mejorar la práctica educativa con la utilizacion de recursos tecnológicos que nos ofrece las TIC (Morales, 2013).

Por otro lado, (Castro-Mujica, 2015) en su tesis "Aplicación de las TIC en el Proceso de Enseñanza Aprendizaje" los docentes en su mayoria poseen título profesional orientado a la docencia cononcen de pedagogia pero son pocos quienes la aplican, en muchos casos estan desactualizados en metodologias, presentan algunas debilidades al implementar el uso de las TIC, en su práctica docente, creando la necesidad de buscar nuevas estrategias metodólogicas que le ayuden a implementa todos estos recursos tecnologicos en las aulas.

El presente trabajo está orientado al análisis de la transición que ha tenido la educación debido a la emergencia sanitaria que atravesó el país a consecuencia de la pandemia Covid-19, la misma que obligó al Ministerio de Educación del Ecuador a disponer "Que, mediante Acuerdo Ministerial No. MINEDUC-MINEDUC-2020-00014-A de 15 de marzo de 2020", la suspensión de clases en todo el territorio nacional; y, la continuidad de labores para todo el personal administrativo y docente del Sistema Nacional de Educación bajo la modalidad de teletrabajo, en virtud de la declaratoria de emergencia sanitaria en el país. lo que ha generado la necesidad no sólo para el desarrollo de competencias en general, sino además para incluir los espacios virtuales de aprendizaje 


\section{Cristian Paúl Espinoza-Espinoza; Darwin Gabriel García-Herrera; Cristian Andrés Erazo-Álvarez;} Juan Carlos Erazo-Álvarez

que nos ofrece el internet y a la vez nos plantee una nueva propuesta como es una educación en red (Ministerio de Educacion, 2020).

Podemos decir que el internet es una red en donde se encuentran interconectadas con varios dispositivos móviles, computadoras, etc. a nivel, local, regional, mundial los mismos que facilitan el compartir información de todo tipo, que se interrelacionan entre sí a través de la utilización e implementación de un lenguaje universal, siendo el internet un sistema de conexiones, a través del cual la gente se comunica de forma descentralizada, formando parte esencial para el desarrollo de nuestra sociedad, siendo fuente de información e investigación para algunos, u ocio y trabajo para otros, brindando espacios virtuales de diferentes maneras, ofreciendo a toda persona una accesibilidad a través de diferentes dispositivos a cualquier hora y desde cualquier lugar.

El internet se ha convertido en una herramienta indispensable en la comunicación, en el entretenimiento y aún más en la educación. Este avance y desarrollo de tecnologías de información y comunicación crean nuevas formas y estilos de aprendizaje, estos a su vez la forma de dar y recibir clases por lo que están ocasionando una evolución el aprendizaje y la educación (Mejía-Salazar \& Gómez-Álvarez, 2017).

Esta evolución ha cambiado la perspectiva de la función de los docentes al involucrarlos en esta sociedad del conocimiento, el internet proporciona un sin número de información en ámbito de la educación y hace revalorar cual es el desempeño y adaptación en el rol del docente ante esta nueva generación de estudiantes, están realmente preparados como docentes ante estos nuevos retos, en donde la tecnología y la información es el pilar fundamental para el desarrollo y crecimiento de esta sociedad.

El objetivo principal que refleja la evolución tecnológica, es proporcionar diversas herramientas, recursos para mejorar la calidad de vida, mediante el uso y aplicación en la información, comunicación y educación; facilitando su accesibilidad, conectividad, manejo y funcionalidad, sin embargo, en la sociedad esta idea no siempre va orientada 
de esta manera, debido a diversos intereses de algunos particulares que utilizan la tecnología para otros fines. (García-Ávila, 2017).

Este avance de la tecnología ha cambiado la realidad de la sociedad, ofreciéndoles múltiples ventajas en diferentes ámbitos, creando una nueva era en el aspecto digital, el mismo que se ha venido desarrollando con el uso e implementación del internet y sus recursos. Con la implementación de las TIC se produce una necesidad de incorporación estrategias, métodos y herramientas para la enseñanza aprendizaje en este nuevo escenario educativo, el mismo que ha obligado a los docentes a incorporar la tecnología como instrumentos fundamentales para lograr el aprendizaje (Colectivo educación infantil y TIC, 2014).

Esta nueva propuesta para la educación en la era digital se basa el aprovechamiento de los recursos que nos brinda el internet y la tecnología, la integración de todos estos medios para mejorar la educación tiene grandes obstáculos que resolver, la escasa formación tecnológica, las prácticas educativas tradicionales tanto de docentes y estudiantes, la disponibilidad y acceso limitado del internet en el sistema educativo ecuatoriano, docentes que por su naturaleza y resistentes a innovar sin compromiso de participar en procesos de capacitación continua en educación, son los problemas referentes a esta situación. Pero es evidente también que el cambio se está produciendo a fuerza, la misma se genera en la práctica, hoy en día estos se producen en la base a las necesidades de los sistemas educativos, la mayoría de docentes se siente obligados a utilizar las tecnologías para poder realizar su trabajo aun sin tener un conocimiento debido.

La pedagogía del ciberespacio como una orientación a la práctica docente constituye una nueva teoría de enseñanza y aprendizaje dentro de la educación virtual. Esta teoría abarca un sistema con ideas, hipótesis y fundamentos científicos y académicos que permiten orientar adecuadamente el trabajo del docente en los espacios virtuales. Desde esta perspectiva la pedagogía del ciberespacio forma parte de una nueva orientación 
pedagógica considerada para la modalidad de estudios virtuales, en donde el proceso de aprendizaje por sus características puede ser asincrónico, es un tipo de formación educativa no presencial, la misma que es producida dentro de los entornos virtuales de aprendizaje y fundamentada en las nuevas tecnologías de la información y la comunicación para su implementación en el proceso de enseñanza y aprendizaje (Hermann-Acosta, 2011).

Es el ciberespacio el escenario principal donde se hace posible la interacción social, cultural y educativa, igual que el Conectivismo la propuesta está centrada en la inclusión de las tecnologías web como parte de la propia actividad cognitiva para aprender y conocer (Solórzano-Martínez \& García-Martínez, 2016). Existen aspiraciones como el de implementar la pedagogía del ciberespacio para explotar al máximo las ventajas que ofrecen las TIC en sectores como la educación, para ello es necesario involucrarse en el ámbito digital orientado a la formación y actualización del docente.

Los docentes están ya involucrados en el proceso de la era digital en una sociedad tecnologizada en donde el día a día y los estilos de vida se han visto intervenidos, comprometidos y transformados por el desarrollo constante e imparable de las tecnologías e internet. Estas tecnologías utilizadas en diferentes espacios virtuales son herramientas que han permitido nuevas formas de comunicarnos, de trabajar, de informarnos, de divertirnos, de participar y vivir en sociedad, en el ámbito educación el rol que desempeña el docente constituye un índice de análisis para el éxito o fracaso en una educación digital ya que constituye el eje de reflexión de este trabajo.

Dicha reflexión apunta a la adecuación del contenido que se va trabajar en proceso de enseñanza aprendizaje, como de las metodologías de enseñanza, que son el reto necesario, e incluso urgente en el contexto social, ya que tal tecnología avanza día a día a su vez la forma de educar está en un cambio de manera constante, en donde los jóvenes están rodeados desde su nacimiento de aparatos tecnológicos y van adquiriendo 
rasgos diferenciados a cualquier otra generación, por lo tanto el rol del docentes es mantenerse actualizado (Viñals-Blanco \& Cuenca-Amigo, 2016).

El proceso de alfabetización digital entre docentes es uno de los problemas que tiene nuestro entorno educativo, ya que todavía existe algunas diferencias y desigualdades sociales que afecta este proceso, no todas las instituciones educativas a nivel nacional cuentan con el acceso a internet recursos tecnológicos para desarrollar sus clases apoyándose en la tecnología. La mayoría de los docentes son conscientes de la importancia de la incorporación de las tecnologías de información y comunicación, conocen de pedagogía, pero son pocos quienes la aplican en proceso de enseñanza y aprendizaje, que involucra la educación actual.

El problema en este tiempo es que el docente no puede incorporar las TIC y adaptarlas a su contexto educativo, en otras palabras, parecería que existe una gran brecha digital entre los estudiantes y los docentes, en el sentido que los estudiantes utilizan la tecnología para participar en redes sociales, interactuar virtualmente con varias personas, para informarse, producir contenidos en diferentes formatos, según sus gustos e intereses; mientras que los docentes generalmente lo consideran a esta TIC como una herramienta de consulta y de comunicación sincrónica.

Esta situación resulta preocupante debido que un gran número de docentes no están incorporando las herramientas digitales en sus actividades académicas, por un lado, desfavorece una mayor interacción, compromiso, creatividad, pensamiento crítico y participación colaborativa en la web y, por el otro, impide que los estudiantes se integren eficientemente a la sociedad actual en donde el uso de las TIC son fundamentales para la apropiación y creación del conocimiento, la comunicación, la innovación, la comercialización (Arrieta \& Montes, 2011).

La pedagogía del ciberespacio permite crear una ruptura de las relaciones de poder entre docentes y estudiantes dentro del aula, en donde su rol debe ser replanteado en donde el estudiante pasa a ser el centro de construcción del aprendizaje y el docente en el guía 


\section{Cristian Paúl Espinoza-Espinoza; Darwin Gabriel García-Herrera; Cristian Andrés Erazo-Álvarez;} Juan Carlos Erazo-Álvarez

y mediador pedagógico para alcanzar los objetivos educativos considerando el avance tecnológico para implementación en la educación (Hermann-Acosta, 2011).

La función del ejercicio docente puede lograr la trasformación de la sociedad de nuestro tiempo ya que el rol del docente exige un alto grado de compromiso dedicación y esfuerzo hacia ellos mismos y a la comunidad educativa en general, exige una opción de vida y una jerarquía de valores orientados a lo humano para la construcción de una sociedad más justa colaboradora y equitativa, para ello el docente deberá actualizarse continuamente, para así contribuir significativamente en el desarrollo personal y social de las nuevas generaciones, que bajo las circunstancias de hoy en día está en un aprendizaje constante.

El rol que desempeña el docente en esta época es de un agente de constante cambio que entiende, promueve, orienta y da sentido al cambio inevitable que trasforma a todos, lo que se exige a los docentes es su compromiso; con la superación personal, con el aprendizaje, con los estudiantes, con la sociedad, con la revolución educativa en la era digital.

\section{METODOLOGÍA}

El presente trabajo investigativo orientado a la pedagogía del ciberespacio, un reto para los docentes, la metodologia utilizada es de carácter no experimental con un enfoque metodologico mixto y de cohorte transversal, dicha investigacion se aplicó a una muestra poblacional que corresponde a 20 docentes y 3 autoridades de la Unidad Educativa "Jadán" que es una institucion fiscal perteneciente a la zona rural del Canton Gualaceo Provincia del Azuay.

Las tecnicas utilizadas fueron una entrevista aplicada a las autoridades mediante una secion a través de la plataforma zoom, la misma que fue aprobada a traves del método Delfi, y una encuesta realizada en la aplicación de google forms, la misma que fue 
EPISTEME KOINONIA

Revista Electrónica de Ciencias de la Educación, Humanidades, Artes y Bellas Artes

Año III. Vol III. $\mathrm{N}^{\circ} 1$. Edición Especial. 2020

Hecho el depósito de Ley: FA2018000022

ISSN: 2665-0282

FUNDACIÓN KOINONIA (F.K).

Santa Ana de Coro, Venezuela

Cristian Paúl Espinoza-Espinoza; Darwin Gabriel García-Herrera; Cristian Andrés Erazo-Álvarez; Juan Carlos Erazo-Álvarez

aplicada a los docentes mediante un lik enviado por whatshapp, esta encueta fue validada por un analisis de fiabilidad con un valor Alfa de Cronbach de 0,985

La información cualitativa fue analizada mediante análisis de contenido documental, estructurandose unidades de análisis y categorías, mientras que la cuantitativo, se procesó en razón de la estadistica descriptiva y chi cuadrado de Pearson.

\section{RESULTADOS}

Se presentan los resultados de la investigación:

\section{Tabla 1.}

Analisis cualitativo de la entrevista.

\begin{tabular}{|c|c|c|}
\hline Unidad de analisis & Categoria & Segmento \\
\hline $\begin{array}{l}\text { Problemas en la transición que de } \\
\text { una educación presencial a una } \\
\text { educación de forma virtual. }\end{array}$ & Problemas en la educacion actual & $\begin{array}{l}\text { - Desconocimiento del } \\
\text { manejo de TIC por parte de } \\
\text { los docentes y alumnos } \\
\text { - Necesidad de capacitacion } \\
\text { docente }\end{array}$ \\
\hline $\begin{array}{l}\text { Implementación de las TIC es en la } \\
\text { educación actual. }\end{array}$ & TIC en la educacion actual & $\begin{array}{l}\text { - Una necesidad } \\
\text { - Prioridad }\end{array}$ \\
\hline $\begin{array}{l}\text { los docentes tienen las } \\
\text { competencias pedagógicas } \\
\text { necesarias para laborar de forma } \\
\text { virtual. }\end{array}$ & El docente en la educacion virtual & $\begin{array}{l}\text { - Existe desconocimiento de } \\
\text { metodologias } \\
\text { - } \quad \text { Falta de capacitación }\end{array}$ \\
\hline $\begin{array}{l}\text { La pedagogía del ciberespacio sería } \\
\text { una alternativa de orientación a la } \\
\text { práctica docente en la actualidad. }\end{array}$ & $\begin{array}{l}\text { La pedagogía del ciberespacio una } \\
\text { alternativa en la educación actual }\end{array}$ & $\begin{array}{l}\text { - Adquisicion de } \\
\text { competencias pedagogícas } \\
\text { para el uso y manejo de los } \\
\text { recursos digitales }\end{array}$ \\
\hline $\begin{array}{l}\text { Las capacitaciones por parte del } \\
\text { ministerio de educación sobre el uso } \\
\text { y manejo de espacios virtuales. }\end{array}$ & $\begin{array}{l}\text { MINEDUC y la capacitacion a los } \\
\text { docentes }\end{array}$ & $\begin{array}{l}\text { - Priorizar las capacitaciones } \\
\text { a la practica docente. }\end{array}$ \\
\hline
\end{tabular}

Fuente: Elaboración propia. 
Como podemos observar en la tabla el analisis de la entrevista a las autoridades de la institucion educativa quienes manifestaron que durante esta transicion de una educacion presencial a una virtual se dieron varios uno de los problemas mas significativos fue el desconocimiento en el uso y manejo de las TIC por parte del cuerpo docente, ademas que estos recursos tecnologicos se han convertido en una necesidad para la educacion actual, en donde la pedagogia debe estar presente como una necesidad primordial que ayudea a orientar todos estos recursos tecnologicos a la educacion.

\section{Tabla 2}

Inconvenientes causados con el cambio de una educación presencial a una educación virtual

\begin{tabular}{cccccc}
\hline & Frecuencia & Porcentaje & $\begin{array}{c}\text { Porcentaje } \\
\text { válido }\end{array}$ & $\begin{array}{c}\text { Porcentaje } \\
\text { acumulado }\end{array}$ \\
\hline \multirow{3}{*}{ Válidos } & Muy en desacuerdo & 4 & 20,0 & 20,0 & 20,0 \\
& En desacuerdo & 2 & 10,0 & 10,0 & 30,0 \\
& De acuerdo & 6 & 30,0 & 30,0 & 60,0 \\
& Muy de acuerdo & 8 & 40,0 & 40,0 & 100,0 \\
& Total & $\mathbf{2 0}$ & $\mathbf{1 0 0 , 0}$ & $\mathbf{1 0 0 , 0}$ & \\
\hline
\end{tabular}

Fuente: Elaboración propia.

Como podemos observar el resultado de la tabla, que en $70 \%$ de los encuestados están de acuerdo que se han producido inconvenientes en el aspecto educativo debido al cambio de una educación presencial a una educación de forma virtual. 
Cristian Paúl Espinoza-Espinoza; Darwin Gabriel García-Herrera; Cristian Andrés Erazo-Álvarez; Juan Carlos Erazo-Álvarez

\section{Tabla 3}

Las TIC se han convertido en una herramienta digital necesaria para el aprendizaje.

\begin{tabular}{cccccc}
\hline & Frecuencia & Porcentaje & $\begin{array}{c}\text { Porcentaje } \\
\text { válido }\end{array}$ & $\begin{array}{c}\text { Porcentaje } \\
\text { acumulado }\end{array}$ \\
\hline \multirow{3}{*}{ Válidos } & Muy en desacuerdo & 3 & 15,0 & 15,0 & 15,0 \\
& En desacuerdo & 2 & 10,0 & 10,0 & 25,0 \\
& De acuerdo & 7 & 35,0 & 35,0 & 60,0 \\
& Muy de acuerdo & 8 & 40,0 & 40,0 & 100,0 \\
\cline { 2 - 6 } & Total & $\mathbf{2 0}$ & $\mathbf{1 0 0 , 0}$ & $\mathbf{1 0 0 , 0}$ & \\
\hline
\end{tabular}

Fuente: Elaboración propia.

Esta tabla refleja un resultado $75 \%$ de los encuestados manifiesta que están de acuerdo en que las TIC se han convertido en una herramienta digital necesaria para el aprendizaje.

\section{Tabla 4.}

El docente además de adquirir competencias de TIC necesita competencias pedagógicas para orientarlas de mejor manera a las necesidades de la educación.

\begin{tabular}{cccccc}
\hline & Frecuencia & Porcentaje & $\begin{array}{c}\text { Porcentaje } \\
\text { válido }\end{array}$ & $\begin{array}{c}\text { Porcentaje } \\
\text { acumulado }\end{array}$ \\
\hline \multirow{3}{*}{ Válidos } & Muy en desacuerdo & 3 & 15,0 & 15,0 & 15,0 \\
& En desacuerdo & 2 & 10,0 & 10,0 & 25,0 \\
& De acuerdo & 7 & 35,0 & 35,0 & 60,0 \\
& Muy de acuerdo & 8 & 40,0 & 40,0 & 100,0 \\
\cline { 2 - 6 } & Total & $\mathbf{2 0}$ & $\mathbf{1 0 0 , 0}$ & $\mathbf{1 0 0 , 0}$ & \\
\hline
\end{tabular}

Fuente: Elaboración propia. 
Cristian Paúl Espinoza-Espinoza; Darwin Gabriel García-Herrera; Cristian Andrés Erazo-Álvarez; Juan Carlos Erazo-Álvarez

Al observa los resultados se puede decir que el $75 \%$ de los docentes encuestados están de acuerdo en que existe la necesidad de incorporar competencias pedagógicas que les ayuden a orientar de mejor manera las TIC dentro del proceso de educación.

\section{Tabla 5.}

Relación entre el cambio de una educación presencial a una educación virtual ha causado inconvenientes en el aspecto educativo y las TIC se han convertido en una herramienta digital necesaria para el aprendizaje.

\begin{tabular}{|c|c|c|c|c|c|c|}
\hline & & \multicolumn{5}{|c|}{$\begin{array}{l}\text { Las TIC se han convertido en una herramienta digital } \\
\text { necesaria para el aprendizaje }\end{array}$} \\
\hline & & $\begin{array}{c}\text { Muy en } \\
\text { desacuerdo }\end{array}$ & $\begin{array}{c}\text { En } \\
\text { desacuerdo }\end{array}$ & De acuerdo & $\begin{array}{l}\text { Muy de } \\
\text { acuerdo }\end{array}$ & Total \\
\hline $\begin{array}{l}\text { El cambio de una } \\
\text { educación presencial a }\end{array}$ & $\begin{array}{c}\text { Muy en } \\
\text { desacuerdo }\end{array}$ & 3 & 1 & 0 & 0 & 4 \\
\hline una educación virtual & En desacuerdo & 0 & 1 & 1 & 0 & 2 \\
\hline ha causado & De acuerdo & 0 & 0 & 4 & 2 & 6 \\
\hline $\begin{array}{l}\text { inconvenientes en el } \\
\text { aspecto educativo }\end{array}$ & Muy de acuerdo & 0 & 0 & 2 & 6 & 8 \\
\hline \multicolumn{2}{|l|}{ Total } & 3 & 2 & 7 & 8 & 20 \\
\hline \multicolumn{2}{|l|}{ Pruebas de chi-cuadrado } & \multicolumn{2}{|c|}{ Valor } & $\mathrm{gl}$ & \multicolumn{2}{|c|}{ Sig. asintótica (bilateral) } \\
\hline \multicolumn{2}{|l|}{ Chi-cuadrado de Pearson } & \multicolumn{2}{|c|}{$25,893^{a}$} & 9 & \multicolumn{2}{|c|}{0,002} \\
\hline \multicolumn{2}{|l|}{ Razón de verosimilitudes } & \multicolumn{2}{|c|}{26,044} & 9 & \multicolumn{2}{|c|}{0,002} \\
\hline \multicolumn{2}{|l|}{ Asociación lineal por lineal } & \multicolumn{2}{|c|}{15,676} & 1 & \multicolumn{2}{|c|}{0,000} \\
\hline $\mathrm{N}$ de casos válidos & & \multicolumn{2}{|l|}{20} & & & \\
\hline
\end{tabular}

a. 16 casillas $(100,0 \%)$ tienen una frecuencia esperada inferior a 5 . La frecuencia mínima esperada es, 20 .

Fuente: Elaboración propia.

En la tabla se puede observar que existe una relación estadísticamente significativa entre las variables, podemos decir que el cambio de la educación presencial a una educación de forma virtual crea la necesidad de la aplicación de las TIC como una herramienta digital necesaria para el aprendizaje en la actualidad. 
Cristian Paúl Espinoza-Espinoza; Darwin Gabriel García-Herrera; Cristian Andrés Erazo-Álvarez; Juan Carlos Erazo-Álvarez

\section{Tabla 6.}

Relación entre un proceso de alfabetización digital al personal docente ayudaría a mejorar la forma de dar y recibir clases y el docente además de adquirir competencias de TIC necesita competencias pedagógicas para orientarlas de mejor manera a las necesidades de la educación.

\begin{tabular}{|c|c|c|c|c|c|}
\hline & \multicolumn{5}{|c|}{$\begin{array}{l}\text { El docente además de adquirir competencias de TIC necesita } \\
\text { competencias pedagógicas para orientarlas de mejor manera a las } \\
\text { necesidades de la educación }\end{array}$} \\
\hline & $\begin{array}{c}\text { Muy en } \\
\text { desacuerdo }\end{array}$ & $\begin{array}{c}\text { En } \\
\text { desacuerdo }\end{array}$ & De acuerdo & $\begin{array}{l}\text { Muy de } \\
\text { acuerdo }\end{array}$ & Total \\
\hline $\begin{array}{l}\text { Un proceso de Muy en } \\
\text { alfabetización digital al desacuerdo }\end{array}$ & 2 & 0 & 0 & 0 & 2 \\
\hline docente En desacuerdo & 1 & 2 & 0 & 0 & 3 \\
\hline ayudaría a mejorar la Indiferente & 0 & 0 & 1 & 0 & 1 \\
\hline forma de dar y recibir De acuerdo & 0 & 0 & 4 & 1 & 5 \\
\hline Muy de acuerdo & 0 & 0 & 2 & 7 & 9 \\
\hline Total & 3 & 2 & 7 & 8 & 20 \\
\hline Pruebas de chi-cuadrado & \multicolumn{2}{|l|}{ Valor } & \multicolumn{3}{|c|}{ Sig. asintótica (bilateral) } \\
\hline Chi-cuadrado de Pearson & \multicolumn{2}{|c|}{$36,270^{\mathrm{a}}$} & 12 & \multicolumn{2}{|c|}{0,000} \\
\hline Razón de verosimilitudes & \multicolumn{2}{|c|}{31,593} & 12 & \multicolumn{2}{|c|}{0,002} \\
\hline Asociación lineal por lineal & \multicolumn{2}{|c|}{16,764} & 1 & \multicolumn{2}{|c|}{0,000} \\
\hline $\mathrm{N}$ de casos válidos & 20 & & & & \\
\hline
\end{tabular}

a. 20 casillas $(100,0 \%)$ tienen una frecuencia esperada inferior a 5 . La frecuencia mínima esperada es, 10.

Fuente: Elaboración propia.

En la tabla se puede observar que existe una relación estadísticamente significativa las variables, podemos decir que el docente en la actualidad necesita un proceso de capacitación en alfabetización digital, la misma que debe ir orientada a la pedagogía para uso y manejo de los recursos digitales que mejoren la forma de dar y recibir clases.

La educación ha tenido un gran cambio, lo que ha generado la necesidad de actualización y capacitación docente no sólo para el desarrollo de competencias en general, sino además para incluir la tecnología. Por lo que, resulta fundamental enfocar la capacitación 


\section{Cristian Paúl Espinoza-Espinoza; Darwin Gabriel García-Herrera; Cristian Andrés Erazo-Álvarez;} Juan Carlos Erazo-Álvarez

docente en competencias digitales, con esta nueva propuesta se debe dejar de ser tradicional, haciendo uso de las nuevas tecnologías, recursos y procesos que provoquen distintos resultados. Se propone desarrollar competencias digitales entre docentes, que les facilite el uso de recursos, herramientas tecnológicas para la elaboración de materiales digitales para su implementación dentro de su práctica diaria, y al mismo tiempo, apoyar a la construcción de nuevos ambientes de aprendizaje (Bustos-López \& Gómez-Zermeño, 2018).

Pero no basta con la adquisición de competencias digitales ni con la implementación de recursos multimediales, interactivos y de fácil acceso, no garantiza que sea efectivos para el logro de aprendizajes, es necesario que el docente genere recursos digitales pero en su proceso de producción se debe a partir desde una reflexión pedagógica donde el docente conozca el contexto y la realidad del estudiante, sobre cómo se aprende y que estos construyan aplicando métodos educativos propuestos por la didáctica sobre cómo se enseña. Para producir estos recursos el docente debe conocer ampliamente el tema que se tratará, saber plantear y direccionar el objetivo propuesta para el aprendizaje, saber definir los contenidos según las necesidades de los estudiantes que deben aprender, como van aprender aquí definir los recursos, medios y procedimientos que se utilizarán que para lograr el acercamiento de los estudiantes al objetivo educativo.

\section{PROPUESTA}

La Tecnologia a evolucionado nuestra era y por ende a producido cambios especialmente en el ambito de la educacion,tanto en la forma de dar y de recibir clases, proponemos en este trabajo la implentacion de una metodologia que se adapte a las necesidades de los entornos virtuales de aprendizaje, que abarque las necesidades de estudiantes y docentes. El flipped classroom o aula invertida es nuestra propuesta para dar la vuelta a la clase tradicional, se fundamenta en tres momentos: antes de la clase, durante la clase, despues de la clase. 
Cristian Paúl Espinoza-Espinoza; Darwin Gabriel García-Herrera; Cristian Andrés Erazo-Álvarez; Juan Carlos Erazo-Álvarez

El aula Invertida asigna a los estudiantes resposabilidades para investigar, revisar contenidos e información en diferentes medios virtuales de forma asincronica guiados por las instrucciones del docente previamente planificadas, para que luego puedan resolver las dudas presentadas y trabajar de forma individual o colaborativa.

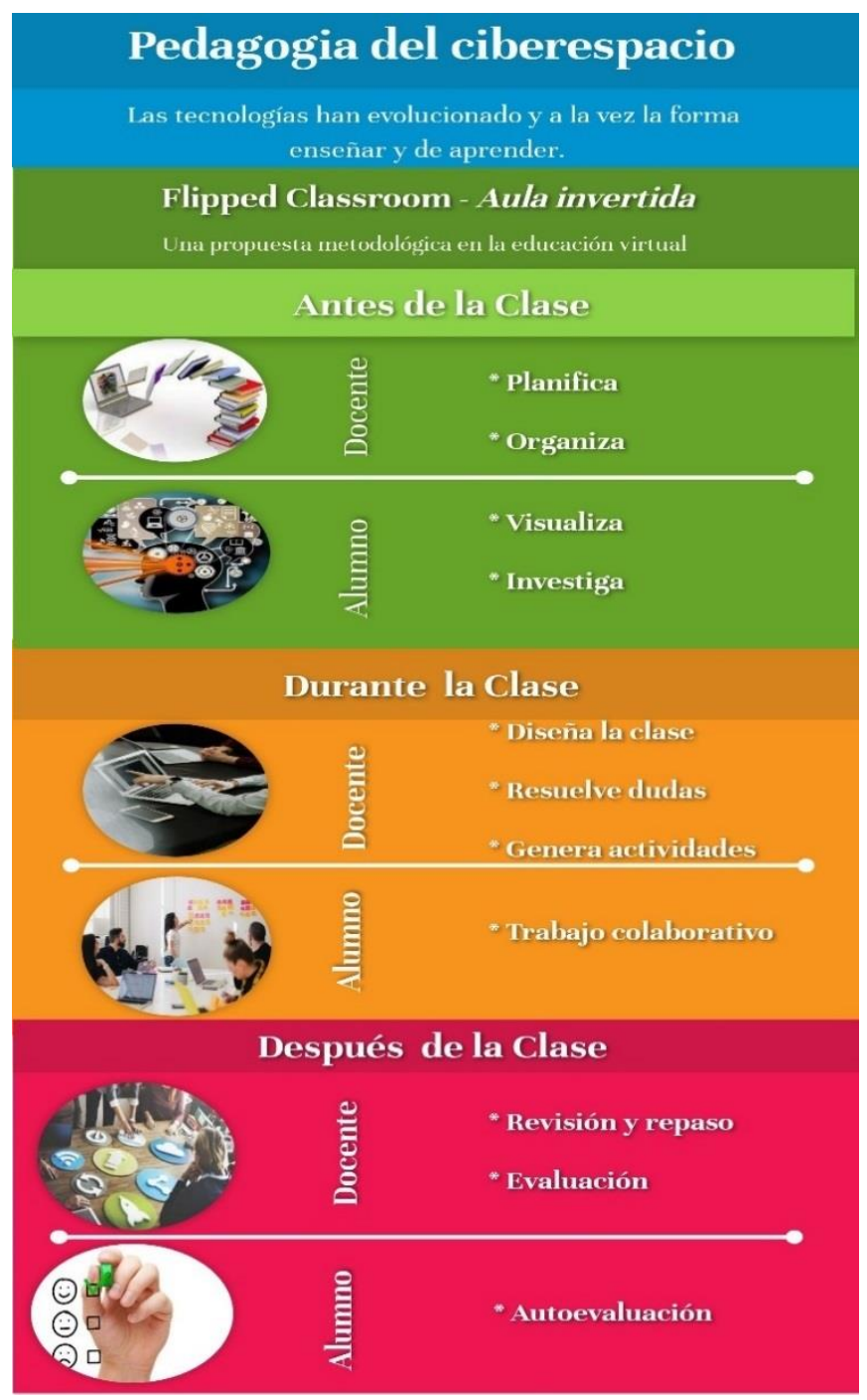

Figura 1. La figura permite observar los pasos para la aplicación del Flipped Classroom. Fuente: Elaboración propia. 


\section{Cristian Paúl Espinoza-Espinoza; Darwin Gabriel García-Herrera; Cristian Andrés Erazo-Álvarez;} Juan Carlos Erazo-Álvarez

\section{Primer momento (Previo a la clase)}

Planificación: el docente deberá elegir el tema planificar en base a los objetivos de aprendizaje y las destrezas que deben desarrollar sus estudiantes basándose en su contexto y en la realidad para la implementación de recursos digitales ya sea videos presentaciones etc.

Organización: Prepara y organiza materiales según las necesidades requeridas de los estudiantes, se pueden utilizar videos de YouTube, Vimeo, o aplicaciones como Edpuzzle, Symbaloo entre otros materiales y recursos para que los estudiantes visualicen, revisen, investigan más a profundidad los conocimientos principales que aborden el tema desde sus hogares.

\section{Segundo momento (Durante la clase)}

Diseñar la clase: el docente diseña y planifica la sesión para la clase según las necesidades de los estudiantes orientándoles y resolviendo dudas que se les haya presentado desarrolla e implementa actividades individuales y colaborativas para generar un aprendizaje activo, se utilizará distintos materiales y recursos para favorecer la comprensión de los conceptos y fomentar la participación.

Actividades de consolidación: Mediante la realización de actividades se debe ir consolidando los conceptos, despejando dudas y reforzando los contenidos. Se debe proponer a cada estudiante o al grupo de estudiantes distintos ejercicios en función de sus necesidades, en donde el docente es el guía y supervisor del trabajo.

Trabajo Colaborativo: El docente debe impulsar el trabajo colaborativo, mediante la utilización de diferentes herramientas digitales como el Google drive, Dropbox, entre otros. En donde los estudiantes puedan compartir y realizar sus trabajos en forma colaborativa en donde puedan elaborar un proyecto, aprender a través de la experimentación. 


\section{Cristian Paúl Espinoza-Espinoza; Darwin Gabriel García-Herrera; Cristian Andrés Erazo-Álvarez;} Juan Carlos Erazo-Álvarez

\section{Tercer momento (Después de la clase)}

Revisión y repaso: El docente revisa el trabajo realizado por sus estudiantes la comparte en clase y ofrece explicaciones, refuerza los contenidos con recursos adicionales, motiva a los estudiantes para profundizar el aprendizaje.

Evaluación y autoevaluación. El docente evalúa el trabajo mediante la aplicación de una rúbrica. Incentiva a la vez a los estudiantes a que se autoevalúen, y evalúen a sus compañeros, para formar un critica constructivista del trabajo planteado con el fin de ir mejorando.

\section{CONCLUSIONES}

Se analizó la transición que se dio en el cambio de una educación presencial a una educación virtual, orientadas a la labor de los docentes en los espacios virtuales de aprendizaje. Esta transición trajo consigo varios cambios en la forma de dar y de recibir clases, causada por el avance de la tecnología como una herramienta necesaria para la aplicación dentro del aula de clase, esta evolución informática y educativa abre un reto a los docentes a capacitarse en la era digital, teniendo en cuenta que un gran número de docentes tiene desconocimiento en el uso de recursos tecnológicos para posterior aplicación en los espacios virtuales en los que están laborando.

La mayoría de los docentes son conscientes de la importancia de la incorporación de las tecnologías de información y comunicación, conocen de pedagogía, pero son pocos quienes la aplican en proceso de enseñanza y aprendizaje, que involucra la educación actual.

Los docentes además de adquirir y adaptar metodologías activas en la enseñanza para el nuevo entorno de aprendizaje tienen que desarrollar habilidades y destrezas digitales que ayuden a incentivar a sus estudiantes a dar un buen uso a la tecnología no solo dentro y fuera de las clases. 
El personal docente igual que sus estudiantes deben aprender de competencias digitales, pero aún más orientarlas de mejor manera a las necesidades que plantea esta nueva sociedad del conocimiento. La propuesta va orientada a la labor del docente a incentivar a una capacitación sobre una alfabetización digital para el manejo de recursos tecnológicos en donde la pedagogía esté orientada a la generación de metodologías activas con el uso de estas tecnologías para el aprendizaje las mismas que deben ser aplicadas dentro de su labor como docente.

\section{FINANCIAMIENTO}

No monetario

\section{AGRADECIMIENTOS}

A la Unidad Educativa “Jadán” del Canton Gualaceo Provincia del Azuay; por apoyar el desarrollo de la investigación.

\section{REFERENCIAS}

Arrieta, A., \& Montes, D. (2011). Alfabetización digital: Uso de las TIC's más allá de una formación instrumentaly una buena infraestructura. [Digital literacy: Use of ICTs beyond an instrumental training and a good infrastructure]. Revista Colombiana de Ciencia Animal, 3(1), 180-197.

Bustos-López, H, \& Gómez-Zermeño, M. (2018). La competencia digital en docentes de preparatoria como medio para la innovación educativa. [Digital competence in high school teachers as a means for educational innovation]. CPU-e. Revista de Investigación Educativa, (26), 66-86.

Castro-Mujica , C. (2015). Aplicación de las TIC en el proceso de enseñanza y aprendizaje de estudiantes con necesidades educativas especiales. [Application of ICT in the teaching and learning process of students with special educational needs]. Guayaquil, Guayas, Ecuador. Recuperado de https://n9.cl/64pif 
Cristian Paúl Espinoza-Espinoza; Darwin Gabriel García-Herrera; Cristian Andrés Erazo-Álvarez; Juan Carlos Erazo-Álvarez

Colectivo educación infantil y TIC. (2014). Recursos educativos digitales para la educación infantil. [Digital educational resources for early childhood education]. Zona Próxima(20), 1-21. Obtenido de https://n9.cl/0698

García-Ávila, S. (2017). Alfabetización Digital. [Digital Literacy]. Razón y Palabra, 21(98),66-81.

Gisbert-Cervera, M., \& Lázaro-Cantabrana, J. (2015). Professional development in teacher digital competence and improving school quality from the teachers' perspective: a case study. Journal of New Approaches in Educational Research, 4(2), 115-122. http://dx.doi.org/10.7821/naer.2015.7.123

Hermann-Acosta, A. (2011). Pedagogia del ciberespacio: hacia la construcción de un conocimiento colectivo. [Cyberspace pedagogy: towards the construction of a collective knowledge]. Sophia, Colección de Filosofía de la Educación(11), 88-103.

Mejía-Salazar, G, \& Gómez-Álvarez, R. (2017). Internet como herramienta didáctica en la formación académica en alumnos de nivel medio superior. [Internet as a didactic tool in academic training in upper secondary level students]. RICSH Revista Iberoamericana de las Ciencias Sociales y Humanísticas, 6(11).

Ministerio de Educacion. (2020). ACUERDO Nro. MINEDUC-MINEDUC-2020-00020-A. Quito: MINEDUC. Recuperado de https://n9.cl/9pfz

Mirete, A. (2010). Formacion docente en TICS. ¿Están losdocentes preparados para la revolución TIC? [Teacher training in ICT. Are teachers ready for the ICT revolution?]. International Journal of Developmental and Educational Psychology, 4(1), 35- 44.

Morales, V. (2013). Desarrollo de competencias digitales docentes en la educación básica. [Development of teaching digital skills in basic education]. Apertura, 5(1), 88-97.

Solórzano-Martínez, F, \& García-Martínez, A. (2016). Fundamentos del aprendizaje en red desde el conectivismo y la teoría de la actividad. [Fundamentals of Networked Learning Based on Connectivism and Activity Theory]. Revista Cubana de Educación Superior, 35(3), 98-112. 
EPISTEME KOINONIA

Revista Electrónica de Ciencias de la Educación, Humanidades, Artes y Bellas Artes

Año III. Vol III. N ${ }^{\circ} 1$. Edición Especial. 2020

Hecho el depósito de Ley: FA2018000022

ISSN: 2665-0282

FUNDACIÓN KOINONIA (F.K).

Santa Ana de Coro, Venezuela

Cristian Paúl Espinoza-Espinoza; Darwin Gabriel García-Herrera; Cristian Andrés Erazo-Álvarez; Juan Carlos Erazo-Álvarez

Viñals-Blanco, A, \& Cuenca-Amigo, J. (2016). El rol del docente en la era digital. [The role of the teacher in the digital age]. Revista Interuniversitaria de Formación del Profesorado, 30(2), 103-114.

(C2020 por el autor. Este artículo es de acceso abierto y distribuido según los términos y condiciones de la licencia CreativeCommons Atribución-NoComercial-Compartirlgual 4.0 Internacional (CC BY-NC-SA 4.0) (https://creativecommons.org/licenses/by-nc-sa/4.0/). 